\title{
AN ANALYSIS OF THE KALMAN, EXTENDED KALMAN, UNCENTED KALMAN AND PARTICLE FILTERS WITH APPLICATION TO DOA TRACKING
}

\author{
Venu Madhava $\mathrm{M}^{1}$, Jagadeesha $\mathrm{S} \mathrm{N}^{2}$, Yerriswamy $\mathrm{T}^{3}$ \\ ${ }^{1}$ Department of Computer Science and Engineering, \\ JNN College of Engineering, Shimoga \\ ${ }^{2}$ Department of Computer Science and Engineering, PESITM, Shimoga \\ ${ }^{3}$ Department of Computer Science and Engineering, \\ KLE Institute of Technology, Hubli
}

\begin{abstract}
Tracking the Direction of Arrival (DOA) Estimation of a multiple moving sources is a significant task which has to be performed in the field of navigation, RADAR, SONAR, Wireless Sensor Networks (WSNs) etc. DOA of the moving source is estimated first, later the estimated DOA using Estimation of Signal Parameters via Rotational Invariance Technique (ESPRIT) is used as an initial value and will be provided to any of the Kalman filter $(K F)$, Extended Kalman filter (EKF), Uncented Kalman filter (UKF) and Particle filter (PF) algorithms to track the moving source based on the motion model governing the motion of the source. ESPRIT algorithm used for the estimation of the DOA is accurate but computationally complex. The present comparative study deals with analysis of tracking the DOA Estimation Of Noncoherent, Narrowband moving sources under different scenarios. The KF (Kalman Filter) is used when the linear motion model corrupted by Gaussian noise, The Extended Kalman Filter (EKF), an approximated and non-linear version of the KF is used whenever the motion model is slightly non-linear but corrupted by Gaussian noise. The process of linearization involves the explicit computation of Jacobian and approximation using Taylor's series is computationally complex and expensive. The computationally complex and expensive procedures of EKF viz explicit computation of Jacobian and approximation using Taylor series are disadvantageous. In order to minimize the disadvantages of EKF are overcomed by the usage of UKF, which uses a transform technique viz. Unscented Transform to linearize the non-linear model corrupted by Gaussian noise and Particle Filter $(P F)$ Algorithms are used when the resultant model is highly non-linear and is corrupted by non-Gaussian noise. Further the literature is concluded with appropriate findings based on the results of the studies of different algorithms in different scenarios carried out.
\end{abstract}

\section{KEYWORDS}

Direction of arrival (DOA), Tracking, Kalman filter, Extended Kalman filter, Uncented Kalman filter, Particle filter.

\section{INTRODUCTION}

The Direction of Arrival (DOA) Estimation and its tracking, is one of the most significant area of array signal processing and finds its applications in the fields of RADAR, SONAR, Wireless

DOI : 10.5121/sipij.2017.8603 
Signal \& Image Processing : An International Journal (SIPIJ) Vol.8, No.6, December 2017

Sensor Networks (WSN),Seismology[1], [2] etc.. Tracking the DOA Estimation is nothing but, estimating the value of DOA of the signals from various moving sources impinging on the array of sensors at each scanning instant of time. The tracking is performed in order to establish correlation among the estimated DOAs at different instants of time. The establishment of correlation between the data (DOA) is also known as data association or estimate association[3]. In the first step, we consider the plane wave fronts from far field are impinging on the Uniform Linear Array (ULA). An appropriate number of snapshots are collected and DOAs are estimated using any of the DOA Estimation algorithms like Multiple SIgnal Classification (MUSIC)[4], Root-MUSIC and Estimation of Signal Parameters via Rotational Invariance Technique (ESPRIT)[5], [6]. The DOA estimation algorithms estimate the DOAs of multiple sources which are stationary but the estimation of the DOAs of the moving sources remain a challenging problem. For estimating DOA of multiple moving sources, the Estimated DOA will be fed as an initial value to tracking algorithms under study. Here, Kalman filter (KF)[7], [8], Extended Kalman filter (EKF)[9], Uncented Kalman filter (UKF)[10] and Particle filters (PF)[11] are considered for study. The algorithm tracking the DOA of multiple moving sources at each scanning instant of time based on their respective target motion models. Unlike, this method, MUSIC, Root-MUSIC[12] or ESPRIT[13] can be used to estimate the instantaneous DOA estimate provided that there is no requirement of data association and the process will be slow. In the present study, a brief comparison among the most used DOA tracking algorithms viz KF, EKF, UKF and PF. It is assumed that the Signals are from non-coherent, narrowband sources. The DOA Estimation and tracking is performed in multiple source scenarios.

\section{BACKGROUND AND FRAMEWORK}

Estimating and tracking the signal parameters viz Time, Frequency, Phase and DOA find significance in areas of RADAR, SONAR, Seismology, Air Traffic Control etc. Various types of estimation techniques such as classical techniques, Beam forming, Spectral based and parametric approaches exist in the literature.

In 1920's Fisher developed a DOA estimation procedure known as Maximum Likelihood (ML) Technique[6], [14], which under the suitable assumptions, estimate the DOA of the incoming signal by maximizing the log likelihood function of the sampled data sequences impinging on the array from a particular direction.

In the beamforming technique, the output power is measured in the direction in which array is steered. Maximum power is observed, if the steered direction is in line with the DOA of the signal .In these types of techniques the output from the array is obtained by combining the data linearly with a weight vector. It is called conventional beamforming technique, if the weight vector used is that of the array.

In subspace based techniques for DOA Estimation such as MUSIC, a spectrum like function of interested parameters are obtained. The distinct peaks of the obtained spectrum are the estimated values of interested parameters. MUSIC algorithm being robust and computationally less complex needs a search algorithm to identify the largest of the peaks. Root-MUSIC \& ESPRIT, being computationally expensive, perform a search over all the values of parameters of interest in order to get more accurate estimates.

For tracking the parameters, adaptive algorithms are used. The adaptive algorithms are in turn divided into two main types; Least Mean Square (LMS) algorithms[9], converge at slower rates depend on the number of step sizes used. Recursive Least Square (RLS)[9] algorithms converge at much higher rates than that of the former type algorithms. The present literature comprises study and analysis of later type algorithms (RLS) Viz Kalman Filter (KF), Extended Kalman 
Signal \& Image Processing : An International Journal (SIPIJ) Vol.8, No.6, December 2017

Filter (EKF), Uncented Kalman Filter (UKF) and Particle Filter (PF)[15] used in tracking the DOA estimation of moving target. The KF algorithms proposed by R.E.Kalman in 1960 considered as basic type of tracking algorithms based on linear state-space model of the moving target. An extension to the KF type of algorithms provided by R. O. Schmidt in 1962 to track parameter which is based on the state-space model which is slightly non-linear. Jacobian and Taylor series approximation is used to linearize the non-linear state model .If the non-linearity increases there is deviation in performance of the EKF. In order to avoid this situation Ulman proposed a new technique which is based on uncented transformation[10] which linearizes highly non-linear state space model and after linearizing the non-linear state models using both techniques, the parameters are tracked using KF algorithms. This section gives out the necessary framework to perform the tracking operation of the optimum DOA Estimate.

\subsection{System model}

Let us consider an ULA of ' $M$ ' identical sensors on which ' $N$ ' narrow band signals are being impinged from the directions $\theta_{1}, \theta_{2}, \theta_{3}, \ldots \ldots \ldots \theta_{N}$. The $i^{\text {th }}$ signal impinging on the array as shown in the Fig 1 is given by

$$
S_{i}(t)=S_{i} e^{j\left(\omega t+\phi_{i}\right)} \quad i=1,2,3, \ldots, N
$$

Where, $S_{i}, \omega, \phi_{i}$ are the amplitude, frequency and phase of the signals of three parameters, phase $\phi$ is considered to be a uniformly distributed random variable. Let us define a column vector $S(t)$ as

$$
S(t)=\left[S_{1}(t), S_{2}(t), \ldots \ldots \ldots S_{N}(t)\right]^{T}
$$

Where ' $T$ ' denotes Transpose.

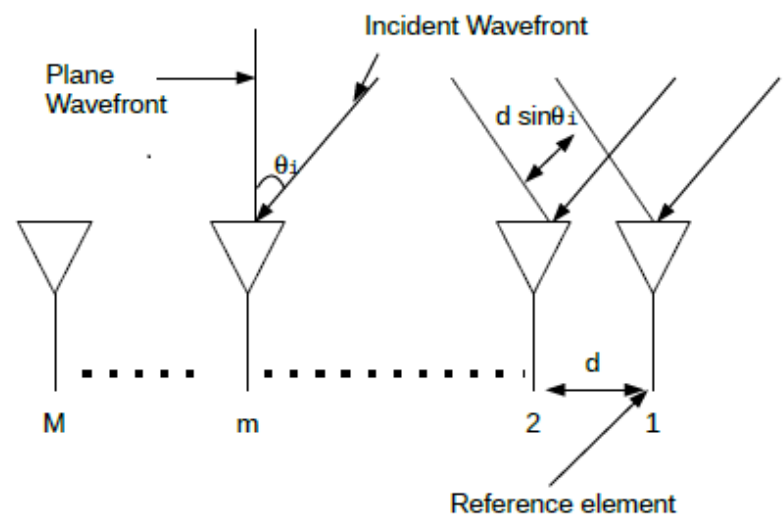

Fig 1: Illustration of the DOA Estimation Model

The direction vector of the $i^{\text {th }}$ source is given by

$$
\begin{gathered}
a\left(\theta_{i}\right)=\left[1, e^{-j \omega_{i} \tau_{1}\left(\theta_{i}\right)}, e^{-j \omega_{i} \tau_{2}\left(\theta_{i}\right)}, \ldots \ldots \ldots \ldots, e^{-j \omega_{(M-1)} \tau_{(M-1)}\left(\theta_{i}\right)}\right]^{T} \quad i=1,2,3 \ldots, N \\
\text { Where, } \tau_{m}=(m-1) \frac{d}{c} \sin \theta_{i} \quad m=1,2,3 \ldots M
\end{gathered}
$$


Signal \& Image Processing : An International Journal (SIPIJ) Vol.8, No.6, December 2017

$d$ is the inter element spacing , $c$ being the propagation velocity of the plane wave front. The inter element spacing is assumed to be less than or equal to half the wavelength of the signal impinging. This assumption is made in order to avoid spatial aliasing.

Substituting (4) in (3) and replacing $\omega=2 \pi f_{c}$, we get the array response vector for the ULA and is given by

$$
a\left(\theta_{i}\right)=\left[1, e^{-j \frac{2 \pi}{\lambda_{\omega}} d \sin \left(\theta_{i}\right)}, \ldots \ldots \ldots \ldots, e^{-j \frac{2 \pi}{\lambda_{\omega}}(M-1) d \sin \left(\theta_{i}\right)}\right]^{T} \quad i=1,2,3 \ldots, N
$$

Where, $f_{c}$ is the carrier frequency and $\lambda_{\omega}$ is the wavelength and they are related by $f_{c}=c / \lambda_{\omega}$

At the $m^{\text {th }}$ element, the received signal is given by

$$
x_{m}(t)=x_{1}(t) \exp \left(-j \frac{2 \pi}{\lambda_{\omega}}(m-1) \frac{d}{c} \sin \left(\theta_{i}\right)\right) \quad i=1,2,3, \ldots, M
$$

Where $x_{I}(t)$ is the received signal vector at the first element of the ULA. The direction vector matrix is given by

$$
A=\left[a\left(\theta_{1}\right), a\left(\theta_{2}\right), \ldots . . a\left(\theta_{N}\right)\right]
$$

Assuming white Gaussian noise $n_{i}(t)$ at all the elements, the received signal at the output of the array is given by,

$$
\begin{aligned}
x(t) & =\left[x_{1}(t), x_{2}(t), x_{3}(t), \ldots . ., x_{M}(t)\right]^{T} \\
& =A S(t)+n(t)
\end{aligned}
$$

Where, $A$ is a $(M x N)$ direction or steering vector matrix. If we discretize the above, the input signals of the array are discrete in time and the output of the array is given by

$$
X(k)=A S(k)+n(k) \quad k=1,2,3, \ldots, K
$$

Where, $k$ is the sample instance and $K$ is the number of snapshots. The parameters of the signal from the source which we are interested in are spatial in nature. Hence they require spatial correlation matrix[16].

$$
\begin{aligned}
& R=E\left\{X(k) X^{H}(k)\right\}=A E\left[S(k) S^{H}(k)\right] A^{H}+\sigma^{2} I \\
& =A R_{S} A^{H}+\sigma^{2} I
\end{aligned}
$$

Where, $R_{s}$ is the signal correlation matrix, $\sigma^{2}$ is the Noise variance and $I$ is the identity matrix.

Practically, the correlation matrix is unknown and it has to be estimated from the array output data. If the underlying processes are ergodic, then the statistical expectation can be replaced by time average.

Let us consider that, $x(k)$ is the signal corrupted by noise having $K$ snapshots are received at the output of the array. The received signal $x(k)$ is denoted by $X$ which is also known as stacked data matrix. The similar stacking is applied to pure signal vector $S(k)$ and the noise vector $n(k)$ as $S$ and $N$ respectively. Equation (9) can be written as 


$$
X=A S+N
$$

Where, $X$ is the received noise corrupted signal matrix of size $(M x K), A$ is the direction or steering vector matrix of size $(M x N), S$ is the signal matrix of size $(N x K)$ and $N$ is the additive white Gaussian matrix of size $(M x K)$.

The ensemble correlation matrix estimate is computed by

$$
\hat{R}=\frac{1}{K} \sum_{k=1}^{K} x(k) x^{H}(k)=\frac{1}{K}\left[X X^{H}\right]
$$

\subsection{Subspace based techniques}

The subspace based methods of DOA estimation use the estimated correlation matrix, decompose it and carry out the analysis on the decomposition. The sub space based technique was invented by V.T. Pisarenko [17], later tremendously progressed by the introduction of MUSIC proposed by R.O.Schmidt [4] and followed by ESPRIT by Roy and Kailath[13]. These techniques use the Eigen decomposition of the estimated correlation matrix into signal and noise subspaces[18], [19].

The array output correlation matrix is given by

$$
\begin{aligned}
\hat{R} & =A E\left[S S^{H}\right] A^{H}+\sigma^{2} I \\
& =A R_{S} A^{H}+\sigma^{2} I
\end{aligned}
$$

Where $R_{s}$ is the signal correlation matrix $\sigma^{2}$ is the noise variance and $I$ is the identity matrix. The correlation matrix of (13) is decomposed using Eigen Value Decomposition (EVD) to obtain

$$
\hat{R}=V \Lambda V^{H}
$$

Where, $V$ is the unitary matrix of Eigen vectors of $R$ as columns, $\Lambda$ is the diagonal matrix of Eigen values of $R$. In the present literature, we assume that the sources are uncorrelated and hence the rank of the correlation matrix $R$ is $\mathrm{M}$ and that of $R_{S}$ is $\mathrm{N}$.

$$
\text { Eigen values of } R: \lambda_{1}>\lambda_{2}>\ldots \ldots>\lambda_{M}
$$

Eigen values of signal subspace $R_{S}: \lambda_{1}>\lambda_{2}>\ldots .>\lambda_{N}$.

Remaining (M-N) Eigen values corresponds to noise subspace. The columns of $\mathrm{V}$ are orthogonal. Hence, the correlation matrix can also be decomposed as

$$
\begin{aligned}
\hat{R} & =V \Lambda V^{H} \\
& =V_{S} \Lambda V_{S}{ }^{H}+V_{n} \Lambda_{n} V_{n}{ }^{H}
\end{aligned}
$$

Where $V_{S}$ are signal Eigen vectors, $\Lambda_{S}$ are signal Eigen values both span the signal subspace $E_{S}$. $V_{n}$ and $\Lambda_{n}$ are noise Eigen vectors and Eigen values respectively spanning the complement of signal subspace called noise subspace $E_{n}$. 
Signal \& Image Processing : An International Journal (SIPIJ) Vol.8, No.6, December 2017

\subsubsection{ESPRIT}

The algorithm is based on the rotational invariance property of the signal subspace. Let us define two sub-matrices, $A_{0} \& A_{1}$ by deleting the first and last columns of steering vectors matrix ' $A$ ' respectively. The matrices $A_{0} \& A_{l}$ are related by the following equation

$$
A_{1}=A_{0} \phi
$$

Where, $\phi$ is the diagonal matrix having roots on its diagonal. Here the DOA Estimation problem gets reduced to finding the matrix $\phi$. Considering the correlation matrix $R$ from eq (12), let us obtain matrices $R_{0}$ and $R_{l}$ by deleting first and last columns of $R$ respectively.

Let us define two matrices $V_{S O}$ which has ' $N$ ' largest Eigen vectors of $R_{0}$ as its columns and similarly $V_{S I}$ is a matrix which is having ' $N$ ' largest Eigen vectors of $R_{l}$ as its columns. The two matrices $V_{S O}$ and $V_{S I}$ are related by a unique non-singular matrix ' $\psi$ ' given by

$$
V_{S 1}=V_{S 0} \Psi
$$

The same two matrices $V_{S O}$ and $V_{S I}$ are related to the steering vector matrix by a non-singular transformation matrix $\Gamma$ with the help of equations given below

$$
V_{S 0}=A_{0} \Gamma \text { and } V_{S 1}=A_{1} \Gamma=A_{0} \phi \Gamma
$$

Substituting eq(16) in (18) we get

$$
V_{S 0} \Psi=A_{0} \phi \Gamma \text { and } A_{0} \Gamma \Psi=A_{0} \phi \Gamma
$$

$\phi$ is estimated using least square problem, the diagonal elements of $\phi$ are the estimates of the

\begin{tabular}{|c|c|c|}
\hline \multicolumn{3}{|c|}{ Input to the algorithm $\theta, a(\theta)$} \\
\hline No & Operation performed & Complexity \\
\hline 1 & Estimation of Correlation Matrix & $K M^{2}$ \\
\hline 2 & Eigen decomposition of correlation Matrix & $O\left(M^{3}\right)$ \\
\hline 3 & $\begin{array}{l}\text { Finding the signal subspace } \\
\text { Forming the } V_{S O} \text { by deleting } 1^{\text {st }} \text { column of } V_{S} \\
\text { Forming the } V_{S I} \text { by deleting last column of } V_{S}\end{array}$ & $O\left(N^{3}\right)$ \\
\hline 4 & Using Least squares solve the resulting equation & \\
\hline 5 & Obtain estimate of $(M x M)$ Matrix $\psi$ & \\
\hline & Total & $K M^{2}+O\left(M^{3}\right)+O\left(N^{3}\right)$ \\
\hline
\end{tabular}
polynomial. The DOA is obtained using (19). The algorithm, along with the number of operations needed is summarized in the table below

Table I Summary of Operation of ESPRIT Algorithm

It has been observed from Table 1 that, the ESPRIT algorithm is having the computational complexity of the order of ' $M$ ' as well as ' $N^{3}$ '. By this we can say that this algorithm is computationally most complex of the three algorithms which are considered for the study. 


\subsection{Tracking the DOA}

The Kalman filter (KF) algorithm proposed by R.E.Kalman is considered as the basic of tracking algorithms used in optimum filtering of non-stationary signals. KF algorithm, also known as dynamic filtering algorithm, is considered as an advantage over the Weiner filter, which fails to address the issue of non-stationarity. In DOA tracking we use KF filter to track the optimum DOA estimate. The estimated DOA using ESPRIT will act as an initial estimate to the Kalman Filter algorithm. Based on the physical model, the algorithm starts tracking the DOA Estimate. The KF algorithm is illustrated using the following steps.

\subsubsection{Tracking model for $\mathrm{KF}$}

Let us consider $\theta_{i}(t), \dot{\theta}_{i}(k), \ddot{\theta}_{i}(k) ; i=1,2,3 \ldots . . q$ gives us the DOA, Angular velocity and angular acceleration of the ' $q$ ' number of sources at time $T$. The equations governing the motion of the $i^{\text {th }}$ source are given by

$$
\left[\begin{array}{c}
\theta_{i}(k+1) \\
\dot{\theta}_{i}(k+1) \\
\ddot{\theta}(k+1)
\end{array}\right]=F\left[\begin{array}{c}
\theta_{i}(k) \\
\dot{\theta}_{i}(k) \\
\ddot{\theta}(k)
\end{array}\right]+\left[\begin{array}{c}
\omega_{i 1}(k) \\
\omega_{i 2}(k) \\
\omega_{i 3}(k)
\end{array}\right]
$$

Where Matrix $F$ is given by

$$
F=\left[\left(\begin{array}{ccc}
1 & T & \frac{T^{2}}{2} \\
0 & 1 & T \\
0 & 0 & 1
\end{array}\right)\right]
$$

Where $T$ is the sampling duration and $\omega_{i}(k), i=1,2,3 \ldots . q$ are random process noise responsible for the random disturbances[11]. It is assumed that $\omega_{i}(k)$ is zero mean white Gaussian noise with covariances indicated as follows

$$
Q_{i}=E\left[\omega_{i}(k) \omega_{i}^{T}(k)\right]
$$

In the tracking model illustrated above, we assume that the acceleration remains constant throughout the sampling interval.

\subsubsection{KF Tracking Algorithm}

In the present study, multiple sources are being tracked. The tracking algorithm is illustrated as follows $x_{i}(k)$ is the state of the ' $q$ ' sources at ' $k^{t h}$ ' instant and is given by

$$
x_{i}(k)=\left[\begin{array}{c}
\theta_{i}(k) \\
\dot{\theta}_{i}(k) \\
\ddot{\theta}(k)
\end{array}\right] \quad i=1,2, \ldots, q
$$

Using equation (23) the source motion governing model, we can write (20) as 
Signal \& Image Processing : An International Journal (SIPIJ) Vol.8, No.6, December 2017

$$
x_{i}(k+1)=F x_{i}(k)+\omega_{i}(k)
$$

$\hat{\theta}_{i}(k)$ is the optimum DOA Estimate of $\theta_{i}(k)$ based on the data obtained during the interval $[(k T,(k+1) T]$. Based on this, the measurement equation can be written as

$$
\hat{\theta}_{i}(k)=\theta_{i}(k)+\eta_{i}(k) \quad i=1,2,3, \ldots, q
$$

Using equations (25) and considering the optimum DOA Estimate, a Kalman filter is used to track the source's state estimate. The state estimation is carried out using the following components.

We can rewrite the equation (25) as

$$
\hat{\theta}_{i}(n)=h\left[\begin{array}{c}
\theta_{i}(n) \\
\dot{\theta}_{i}(n) \\
\ddot{\theta}_{i}(n)
\end{array}\right]+\eta_{i}(n) \quad i=1,2,3, \ldots, q
$$

Where $h=[1,0,0]$ since we are going to track only angular position, we neglect the angular velocities and acceleration and hence the ' $h$ ' vector. Using Equations (22-26) the Kalman filter equation can be written as

$$
\left[\begin{array}{c}
\hat{\theta}_{i}(n \mid n) \\
\dot{\theta}_{i}(n \mid n) \\
\ddot{\theta}_{i}(n \mid n)
\end{array}\right]=\left[\begin{array}{c}
\hat{\theta}_{i}(n \mid n-1) \\
\hat{\theta}_{i}(n \mid n-1) \\
\hat{\ddot{\theta}}_{i}(n \mid n-1)
\end{array}\right]+L_{i}(n)\left[\hat{\theta}_{i}(n)-\hat{\theta}_{i}(n \mid n-1)\right]
$$

The first term in the RHS of (27) are predicted estimates, the prediction is carried out using the measurements up to $(n-1) T$.

The predicted state estimates of $\hat{\theta}_{i}(n \mid n), \dot{\theta}_{i}(n \mid n), \ddot{\theta}_{i}(n \mid n)$ are given by $\left[\hat{\theta}_{i}(n \mid n-1), \hat{\dot{\theta}}_{i}(n \mid n-1), \hat{\ddot{\theta}}_{i}(n \mid n-1)\right]$ respectively. The Kalman gain $L_{i}(n)$ acts as a weighted compensator and is given by

$$
L_{i}(n)=\frac{P_{i}(n \mid n-1) h^{T}}{h P(n \mid n-1) h^{T}+J_{i i}^{-1}}
$$

Where, $J_{i i}$ is the $i^{\text {th }}$ element of the Fisher information Matrix.

The Kalman filter recursions are carried out in the following steps

1) In the first interval, ESPRIT is used to find the initial estimate of DOA.

2) In the next step we use the optimum DOA estimate as the initial value and start tracking the DOA Estimates. The Kalman filter algorithm is summarised in Table II 
Signal \& Image Processing : An International Journal (SIPIJ) Vol.8, No.6, December 2017

Table II Summary of KF Algorithm

\begin{tabular}{|l|l|}
\hline No & Operation performed \\
\hline Step 1 & Initialization of the KF Algorithm \\
\hline Step 2 & $\begin{array}{l}\text { Time recursion for } n=1,2,3, \ldots \ldots \\
\text { a)Finding the signal prediction } \\
\text { b) Determination of apriori error covariance } \\
\text { c) Finding the Kalman Gain } \\
\text { d) Finding the Signal Update } \\
\text { e)Determination of aposteriori error covariance }\end{array}$ \\
\hline Step 3 & Obtaining the filtered estimate ( Output) \\
\hline
\end{tabular}

\subsubsection{EKF Tracking Algorithm}

The Extended Kalman filter is considered as a non-linear version of Kalman filter. The Extended Kalman filter receives the non-linear input and acts on its present estimate of mean and covariance to get the output parameter. It is considered as one of the best tool in nonlinear estimation. If the model of the system is non-linear, the usage of KF is extended with the help of linearization procedure known as EKF. The aforementioned extension is possible due to the possibility of representation of KF using differential or difference equations for continuous and discrete systems respectively. Systems represented using non-linear state-space equations are solved using the EKF leading towards approximate solution. The state space equations of a nonlinear system are given by

$$
\begin{aligned}
& x_{i}(n+1)=F\left(n, x_{i}(n)\right)+\omega_{i}(n) \\
& y_{i}(n)=h\left(n, x_{i}(n)\right)+\omega_{i}(n)
\end{aligned}
$$

Where, $\omega_{1}(k)$ and $\omega_{2}(k)$ are uncorrelated zero mean white noise processes with correlation matrices $Q_{1}(n)$ and $Q_{2}(n)$ respectively. In the above equation, the term $F(n, x(n))$ is non-linear transition matrix and $h\left(n, x_{i}(n)\right)$ is non-linear measurement matrices, both of them can also be considered as time varying. In EKF, the state-space model is linearized at each instant of time around the most recent state estimate considered at $\hat{x}_{i}\left(n \mid y_{n}\right)$ and $\hat{x}_{i}\left(n \mid y_{n-1}\right)$ based on the functional considered.

After obtaining the linear model, the KF equations can be applied. The approximation is carried out in two steps which are illustrated below

Step 1: Obtaining the Jacobian of the state transition and measurement matrices.

$$
\begin{gathered}
F(n+1, n)=\left.\frac{\partial F(n, x)}{\partial x}\right|_{x_{i}=\hat{x}_{i}\left(n \mid y_{n}\right)} \\
h(n)=\left.\frac{\partial h\left(n, x_{i}\right)}{\partial x}\right|_{x_{i}=\hat{x}_{i}\left(n \mid y_{n-1}\right)}
\end{gathered}
$$


Signal \& Image Processing : An International Journal (SIPIJ) Vol.8, No.6, December 2017

The $i, j^{\text {th }}$ entry of transition matrix is the partial derivative of the $i^{\text {th }}$ component of $F(n, x(n))$ with respect to $j^{\text {th }}$ component of $x(n)$. Similarly the $i, j^{\text {th }}$ entry of $\mathrm{h}(\mathrm{n})$ is nothing but the partial derivative of $i^{\text {th }}$ component of $h(n, x(n))$ with respect to $j^{\text {th }}$ component $x$. In the case of transition matrix, the derivatives are evaluated at $\hat{x}_{i}\left(n \mid y_{n}\right)$ and in the case of measurement matrix, the derivatives are evaluated at $\hat{x}_{i}\left(n \mid y_{n-1}\right)$.

Step 2: Obtaining the Taylor series approximation of the non-linear state transition and measurement matrices.

After obtaining the $F(n+1, n)$ and $h(n)$, they are written as first order Taylor approximation of non-linear state transition and measurement matrices around $\hat{x}_{i}\left(n \mid y_{n}\right)$ and $\hat{x}_{i}\left(n \mid y_{n-1}\right)$ respectively as

$$
\begin{aligned}
& F(n, x(n)) \square F\left(n, \hat{x}_{i}\left(n \mid y_{n}\right)\right)+F(n+1, n)\left[x_{i}(n)-\hat{x}_{i}\left(n \mid y_{n}\right)\right] \\
& h\left(n, x_{i}(n)\right) \square h\left(n, \hat{x}_{i}\left(n \mid y_{n-1}\right)\right)+h(n)\left[x_{i}(n)-\hat{x}_{i}\left(n \mid y_{n-1}\right)\right]
\end{aligned}
$$

With the help of approximate expressions we can write the approximate version of non-linear state space equations as follows

$$
\begin{aligned}
& x_{i}(n+1)=F(n+1, n) x_{i}(n)+\omega_{1}(n)+d(n) \\
& \bar{y}(n)=h(n) x_{i}(n)+\omega_{2}(n) \\
& \bar{y}(n)=y(n)-\left[h\left(n, \hat{x}_{i}\left(n \mid y_{n-1}\right)\right)-h(n) \hat{x}_{i}\left(n \mid y_{n-1}\right)\right] \\
& \text { and } \\
& d(n)=F\left(n, \hat{x}_{i}\left(n \mid y_{n-1}\right)\right)-F(n+1, n) \hat{x}_{i}\left(n \mid y_{n}\right)
\end{aligned}
$$

From the above equations, $\bar{y}(n)$ contains all the known terms at time ' $n$ ' hence it can be considered as the observation vector at time ' $n$ '. If the system dynamics are linear, then $\bar{y}(n)=y(n)$ and $d(n)=0$.The EKF equations can be applied to the above linearized model as shown below.

$$
\begin{aligned}
& \hat{x}_{i}\left(n+1 \mid y_{n}\right)=F(n+1, n) \hat{x}_{i}\left(n \mid y_{n}\right)+d(n) \\
& =F(n+1, n) \hat{x}_{i}\left(n \mid y_{n}\right)+F\left(n, \hat{x}_{i}\left(n \mid y_{n-1}\right)\right)-F(n+1, n) \hat{x}_{i}\left(n \mid y_{n}\right) \\
& =F\left(n, \hat{x}_{i}\left(n \mid y_{n}\right)\right)
\end{aligned}
$$


Signal \& Image Processing : An International Journal (SIPIJ) Vol.8, No.6, December 2017

$$
\begin{aligned}
& \hat{x}_{i}\left(n \mid y_{n}\right)=\hat{x}_{i}\left(n \mid y_{n-1}\right)+L_{f}(n) \alpha(n) \\
& \alpha(n)=\bar{y}(n)-h(n) \hat{x}_{i}\left(n \mid y_{n-1}\right) \\
& =y(n)-h\left(n, \hat{x}_{i}\left(n \mid y_{n-1}\right)+h(n) \hat{x}_{i}\left(n \mid y_{n-1}\right)-h(n) \hat{x}_{i}\left(n \mid y_{n-1}\right)\right. \\
& =y(n)-h(n) \hat{x}_{i}\left(n \mid y_{n-1}\right)
\end{aligned}
$$

The Procedure explained can be summarized with the help of the following Table III

Table III. Summary of EKF Algorithm

\begin{tabular}{|l|l|}
\hline No & Operation performed \\
\hline Step 1 & $\begin{array}{l}\text { a) Linear approximation of non-linear state transition matrix } \\
\text { b) Linear approximation of non-linear measurement matrix }\end{array}$ \\
\hline Step 2 & Initialization of the KF Algorithm \\
\hline Step 3 & $\begin{array}{l}\text { Time recursion for n=1,2,3,...... } \\
\text { a) Finding the signal prediction } \\
\text { b) Determination of apriori error covariance } \\
\text { c) Finding the Kalman Gain } \\
\text { d) Finding the Signal Update } \\
\text { e) Determination of aposteriori error covariance }\end{array}$ \\
\hline Step 4 & Obtaining the filtered estimate ( Output) \\
\hline
\end{tabular}

\subsubsection{UKF Tracking Algorithm}

The performance of EKF is poor if the state transition and observation models i.e, predict and update functions $f$ and $h$ are highly non-linear. This poor performance is due to the propagation of covariance through the linearization of underlying non-linear model. The UKF locates a minimal set of points known as sigma points around the mean, with the help of a sampling technique known as Uncented transform. A new mean and covariance estimate are formed by non-linear functions, through which the located sigma points are propagated. This results in a new type of filter which estimates true mean and covariance. The present technique overcomes the requirement of explicit computation of Jacobian.

\section{Prediction}

Similar to EKF, the UKF Prediction is obtained independently by the linear combination of UKF update and a linear update. The UKF update is a linear combination obtained from UKF prediction and linear prediction. The mean and covariance of process noise is blended with estimated state and covariance given by

$$
X_{k-1 \mid k-1}^{a}=\left[\hat{X}_{k-1 \mid k-1}^{T} E\left[w_{k}^{T}\right]\right]^{T}
$$


Signal \& Image Processing : An International Journal (SIPIJ) Vol.8, No.6, December 2017

$$
P_{k-1 \mid k-1}^{a}=\left[\begin{array}{cc}
P_{k-11 k-1} & 0 \\
0 & Q_{k}
\end{array}\right]
$$

The augmented state, having dimension $L$ and covariance gives a set of $2 L+1$ sigma points

$$
\begin{aligned}
& \chi_{k-1 k-1}^{0}=X_{k-11 k-1}^{a} \\
& \chi_{k-11 k-1}^{i}=X_{k-11 k-1}^{a}+\left(\sqrt{(L+\lambda) P_{k-1 k-1}^{a}}\right)_{i}, i=1, \ldots ., L \\
& \chi_{k-11 k-1}^{i}=X_{k-11 k-1}^{a}-\left(\sqrt{(L+\lambda) P_{k-1 k-1}^{a}}\right)_{i-L}, i=L+1, \ldots ., 2 L
\end{aligned}
$$

Where

$$
\left(\sqrt{(L+\lambda) P_{k-11 k-1}^{a}}\right)_{i}
$$

is the $i^{\text {th }}$ column of the matrix square root of

$$
(L+\lambda) P_{k-11 k-1}^{a}
$$

using the definition: square root $\mathbf{A}$ of matrix $\mathbf{B}$ satisfies

$$
B=A A^{T}
$$

An efficient and stable method, Cholesky decomposition is used to calculate the square root of the matrix. The transition function $f$ allows the sigma points to propagate through it.

$$
\chi_{k-1 \mid k-1}^{i}=f\left(\chi_{k-1 \mid k-1}^{i}\right), i=0,1,2, \ldots, 2 L
$$

where $f: R^{L} \rightarrow R^{|x|}$. The predicted state and covariance are produced by recombination of weighted sigma points

$$
\begin{aligned}
& \hat{X}_{k \mid k-1}=\sum_{i=0}^{2 L} W_{s}^{i} \chi_{k \mid k-1}^{i} \\
& P_{k \mid k-1}=\sum_{i=0}^{2 L} W_{c}^{i}\left[\chi_{k \mid k-1}^{i}-\hat{X}_{k \mid k-1}\right]\left[\chi_{k \mid k-1}^{i}-\hat{X}_{k \mid k-1}\right]^{T}
\end{aligned}
$$

The covariance and state weights are given by 
Signal \& Image Processing : An International Journal (SIPIJ) Vol.8, No.6, December 2017

$$
\begin{aligned}
& W_{s}^{0}=\frac{\lambda}{L+\lambda} \\
& W_{c}^{0}=\frac{\lambda}{L+\lambda}+\left(1-\alpha^{2}+\beta\right) \\
& W_{s}^{i}=W_{c}^{i}=\frac{1}{2(L+\lambda)} \\
& \lambda=\alpha^{2}(L+\kappa)-L
\end{aligned}
$$

\section{Update}

The augmentation of the predicted state and covariance are carried out as before but now with the mean and covariance of measurement noise.

$$
\begin{aligned}
& X_{k-1 \mid k-1}^{a}=\left[\hat{X}_{k-1 \mid k-1}^{T} E\left[V_{k}^{T}\right]\right]^{T} \\
& P_{k-1 \mid k-1}^{a}=\left[\begin{array}{cc}
P_{k-11 k-1} & 0 \\
0 & R_{k}
\end{array}\right]
\end{aligned}
$$

A set consisting of $2 L+1$ sigma points is obtained by the augmentation of state and covariance. The resulting augmented state is having a dimension of $L$.

$$
\begin{aligned}
& \chi_{k-11 k-1}^{0}=X_{k-11 k-1}^{a} \\
& \chi_{k-11 k-1}^{i}=X_{k-11 k-1}^{a}+\left(\sqrt{(L+\lambda) P_{k-11 k-1}^{a}}\right)_{i}, i=1, \ldots, L \\
& \chi_{k-11 k-1}^{i}=X_{k-11 k-1}^{a}-\left(\sqrt{(L+\lambda) P_{k-11 k-1}^{a}}\right)_{i-L}, i=L+1, \ldots, 2 L
\end{aligned}
$$

The augmented sigma points, used in the UKF prediction is given by the following

$$
\chi_{k \mid k-1}:=\left[\chi_{k \mid k-1}^{T} \quad E\left[v_{k}^{T}\right]\right]^{T} \pm \sqrt{(L+\lambda) R_{k}^{a}}
$$

Where

$$
R_{k}^{a}=\left[\begin{array}{cc}
0 & 0 \\
0 & R_{k}
\end{array}\right]
$$

The observation function $h$ allows the projection of sigma points through it, is given by.

$$
\gamma_{k}^{i}=h\left(\chi_{k \mid k-1}^{i}\right), i=0,1, \ldots, 2 L
$$

The obtained measurement and predicted measurement covariance ,by the recombination of weighted sigma points is given by 
Signal \& Image Processing : An International Journal (SIPIJ) Vol.8, No.6, December 2017

$$
\begin{aligned}
& \hat{z}_{k}=\sum_{i=0}^{2 L} W_{s}^{i} \gamma_{k}^{i} \\
& P_{z_{k} z_{k}}=\sum_{i=0}^{2 L} W_{c}^{i}\left[\gamma_{k}^{i}-\hat{z}_{k}\right]\left[\gamma_{k}^{i}-\hat{z}_{k}\right]^{T}
\end{aligned}
$$

The state-measurement cross-covariance matrix can be used for the computation of Kalman gain in the case of UKF.

$$
\begin{aligned}
& P_{z_{k} z_{k}}=\sum_{i=0}^{2 L} W_{c}^{i}\left[\chi_{k \mid k-1}^{i}-\hat{x}_{k \mid k-1}\right]\left[\gamma_{k}^{i}-\hat{z}_{k}\right]^{T} \\
& K_{k}=P_{x_{k} z_{k}} P_{x_{k} z_{k}}^{-1}
\end{aligned}
$$

Similar to KF, the updated state is the combination of predicted state and innovation processes weighted by Kalman Gain.

$$
\hat{X}_{k \mid k}=\hat{X}_{k \mid k-1}+K_{k}\left(z_{k}-\hat{z}_{k}\right)
$$

The updated covariance is the result of the subtraction of predicted measurement covariance from the predicted covariance weighted by the Kalman Gain is given by.

$$
P_{k \mid k}=P_{k \mid k-1}-K_{k} P_{z_{k} z_{k}} K_{k}^{T}
$$

\subsubsection{PF Tracking}

Del and Moral [20], [21] used the term "Particle filters" with reference to the mean field particle interaction methods in fluid dynamics. Later in Liu and Chen [22] proposed the term "Sequential Monte Carlo". In signal processing and Bayesian statistical interference, the solution to the following problems are given by these particle filters or sequential Monte Carlo methods. When there are random noises in both sensors and dynamics of the system, the estimation of internal states of the dynamics of the system is partial. The usage of PF and sequential Monte Carlo is to compute the posterior distributions of Markov process noise based on noisy and partial observations.

Considering a genetic type mutation selection sampling approach, along with a set of particles are used to represent the posterior distributions of stochastic process from the given noisy/partial observations. The state -space model used here can be non-linear and the initial state and noise distributions may assume any form. Samples from the required distributions are generated without any assumptions about state-space models or distributions.

A set of particles are used to represent the samples from the obtained distribution. A likelihood weight is assigned to the particles used. The assigned likelihood weight represents the particle obtained by sampling the probability density function. The particle filtering algorithms suffer from the issue of weight collapse lead by weight disparity. The issue of weight collapse can be overcome by a procedure of resampling much before the weight become uneven. The solutions to Hidden Markov Chain(HMM)and non-linear filtering is provided by the particle filter methodology. When there are hard non-linearities, numerical methods, based on fixed grid approximations, Markov chain Monte carlo techniques and EKF haven't performed well when the systems are having large scale unstable processes $\{$ cite add $\}$. The primary aim of particle filter 
Signal \& Image Processing : An International Journal (SIPIJ) Vol.8, No.6, December 2017

is to estimate the posterior density of state variables when the observation variables are given. The system consisting of hidden and observable variables of HMM is solved by PF.A known functional form is used to relate the observation process with state process.

Generally, a particle filter carries the estimation of the posterior distribution of hidden states by using the observation or measurement process. The problem of filtering is the sequential estimation of the values of hidden states $X_{k}$ by observing the values obtained by the observation process $Y_{0}, Y_{1} \ldots \ldots Y_{k}$ at all $k$ instants of time. The posterior density $p\left(x_{k} \mid y_{0}, y_{1} \ldots . y_{k}\right)$ is followed by all Bayesian estimates of $X_{k}$. An emperical measure associated with a genetic type particle algorithm is used by the $\mathrm{PF}$ algorithm to provide an approximation of the conditional probabilities. Contrarily, the MCM approach also known as importance sampling approach models the full posterior density $p\left(x_{0}, x_{1}, \ldots . . x_{k} \mid y_{0}, y_{1, \ldots . .} y_{k}\right)$. A generic particle filter estimates the posterior distribution of the hidden states using the observation measurement process. Consider a state-space shown in the diagram below.

\section{The Signal-Observation Model}

Particle filtering methods often assume that $X_{k}$ and the observations $Y_{k}$ can be modeled in this form: A Markov process $X_{0}, X_{1}, \ldots \ldots$ on $\mathrm{R}^{\mathrm{dx}}$ (for some $\mathrm{d}_{\mathrm{x}}>1$ ) is based on transition probability density $p\left(x_{k} \mid x_{k-1}\right)$. The synthetical form of the model with an initial probability density $p\left(x_{0}\right)$ is given by

$$
X_{k} \mid X_{k-1}=x_{k} \square p\left(x_{k} \mid x_{k-1}\right)
$$

The observations $Y_{0}, Y_{l, \ldots,,}$, take values in state-space on $\mathrm{R}^{\mathrm{dy}}$ (for some $\mathrm{d}_{\mathrm{y}}>1$ ) are independent if the $X_{0}, X_{1}, \ldots \ldots$ are known i.e, each $Y_{k}$ only is dependent on $X_{k}$. Additionally, a conditional distribution is assumed for $Y_{k}$ provided that $X_{k}=x_{k}$ are absolutely continuous, synthetically

$$
Y_{k} \mid X_{k}=y_{k} \square p\left(y_{k} \mid x_{k}\right)
$$

The example of a system with the above properties is:

$$
Y_{k}=h\left(X_{k}\right)+V_{k}
$$

In the eq $W_{k}$ and $V_{k}$ are mutually independent sources of known probability density functions. The other functions $g$ and $h$ are also known. The eqns(49),(50) are similar to state space equations of KF. The exact Bayesian filtering distribution is found by KF if the functions $g$ and $h$ in the above equations are linear and $W_{k}$ and $V_{k}$ are Gaussian else the other methods based on the KF i.e, EKF and UKF are used. The assumption of the continuity of initial distribution and Markov chain transitions with respect to Lebesgue method are relaxed. In order to design a PF, It has been assumed that the transitions $X_{k-1} \mid X_{k}$ of the Markov chain $X_{k}$, can be sampled and the likelihood function $x_{k} \mid p\left(y_{k} \mid x_{k}\right)$. The assumptions of absolute continuity of Markov transition $X_{k}$ are used to derive different formulae in between posterior distributions with the help of the Bayes' rule for conditional densities.

\section{RESULTS AND DISCUSSIONS}

The present section deals with the results obtained during the course of analysis. The analysis of tracking the DOA using KF, EKF, UKF and PF. Finally the section will be concluded by the comparative analysis of tracking the DOA using KF,EKF,UKF and PF. The study depicts that, all 
the tracking algorithms considered for study are capable of tracking the DOA Estimation of moving sources under different scenarios.

\section{A. Result of DOA estimation tracking using KF algorithm.}

Fig 2 shows tracking the DOA estimation of multiple targets under the linear motion model. having constant velocity and corrupted by Gaussian noise scenario. The initial values $[-10,0$, 10], are determined using ESPRIT algorithm. Further, the initial values are given to KF for the purpose of tracking. Here, initial value is assumed and the target motion is defined in the process equation. The target motion model assumed here is considered to be linear.
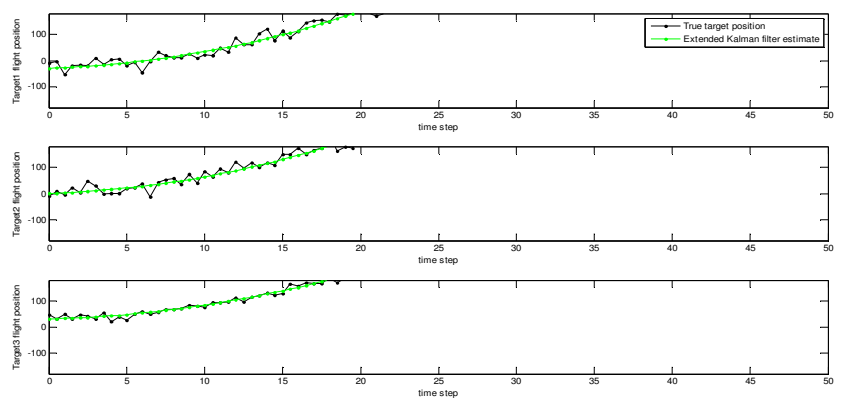

Figure 2 Tracking DOA Estimation using KF algorithm.

\section{B. Result of DOA estimation tracking using EKF algorithm.}

When the model becomes slightly non- linear, the KF fails to track the movement of the targets. At this juncture, an extension to the KF, capable of handling the slight amount of non-linearity is used. The model obtained by the extension of KF is known as EKF, uses Explicit computation of Jacobians and Taylor series approximation technique to linearize the non-linear model. Once the non-linear model gets linearized by the aforementioned process, the KF is used to track the DOA. Fig 3 Shows DOA tracking of multiple targets under the slightly non-linear motion model and corrupted with Gaussian noise scenario. The initial values are estimated with the help of ESPRIT algorithm is assumed to be $[-10,0,10]$ and the performance is analyzed.

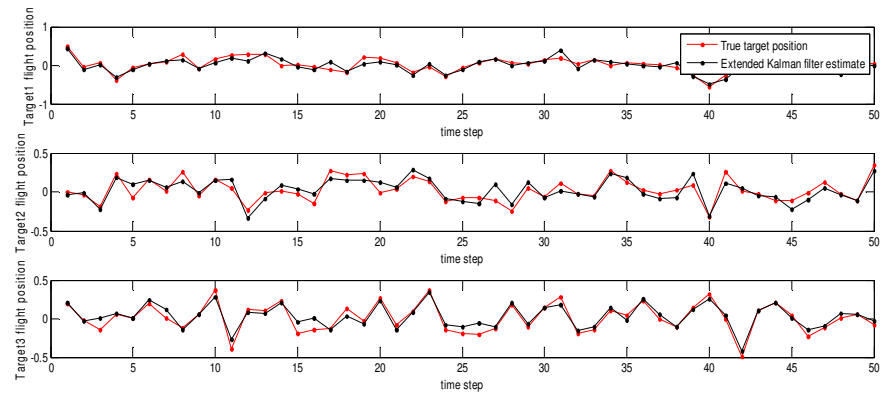

Fig 3 Tracking DOA Estimation using EKF algorithm.

\section{Result of DOA estimation tracking using UKF algorithm.}

The explicit computation of Jacobian and approximation using Taylor series is computationally complex. In order to overcome the difficulty of complexity, a transform technique known as Uncented Transform is used to linearize the non-linear model relatively the computation is less complex compared to the EKF instead of explicit computation of Jacobians. Fig 4 Shows DOA estimation of multiple targets with the help of ESPRIT algorithm is considered as initial value to 
Signal \& Image Processing : An International Journal (SIPIJ) Vol.8, No.6, December 2017

the UKF for the purpose of tracking. Here, $[-10,0,10]$ initial value is assumed, and the performance is analyzed.
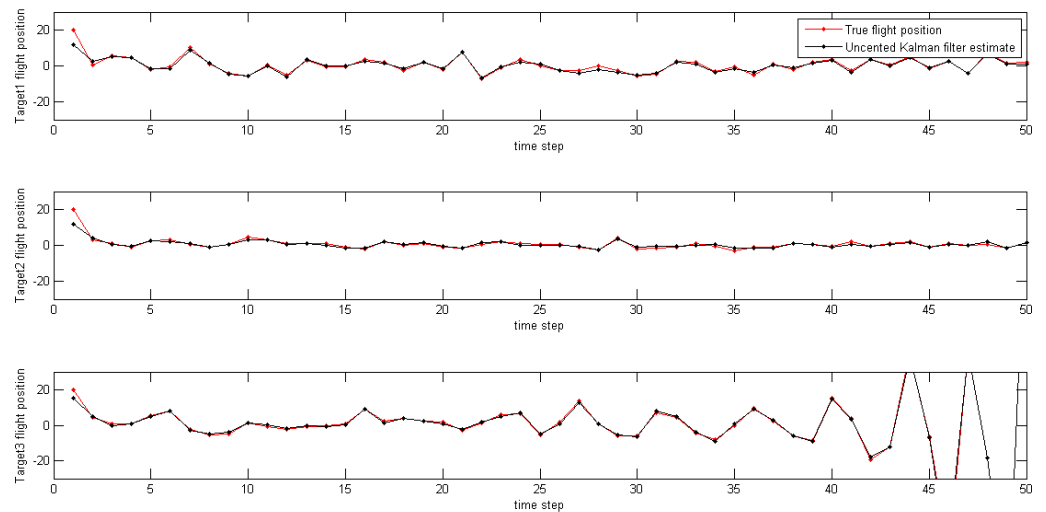

Fig 4 Tracking DOA Estimation using UKF algorithm.

\section{Result of DOA estimation tracking using PF algorithm.}

When the system is completely nonlinear,both EKF and UKF fail to track the DOA estimation of the moving targets.A complete non-linear state estimator know as Particle filter is used to track the DOA estimation of the targets. The performance of PF is more effective compared to EKF and UKF. Fig 5 shows DOA tracking of multiple targets under the non-linear motion model corrupted by non-Gaussian noise scenario. The initial value assumed $[-10,0,10]$ is estimated with the help of ESPRIT algorithm is considered as initial value to the PF for the purpose of tracking and the performance is analyzed.
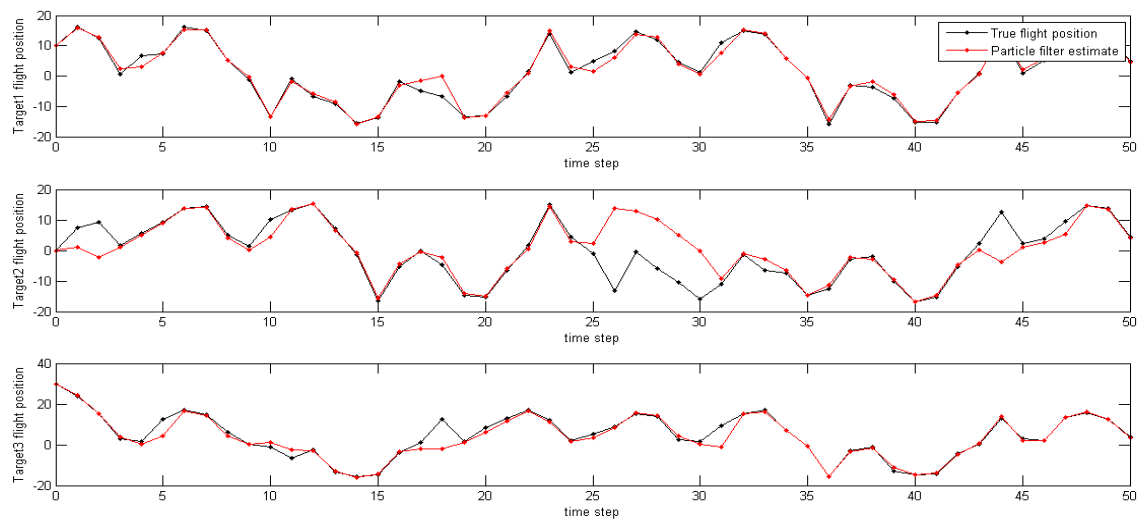

Fig 5 Tracking DOA Estimation using PF algorithm.

\section{CONCLUSIONS}

The present study depicts that, all of the four tracking algorithms are capable of tracking multiple moving targets under different scenarios. When the signal is non-stationary but linear, KF can be used for the purpose of tracking. As the signal gets slightly non-linear, KF fails to track the signals however a suitable extension applied to KF to handle this non-linearity by usage Jacobians and Taylor's series methods to get EKF. But the explicit computation of Jacobians and Taylor's series are computationally expensive and complex. In order to improve the complexity a transform technique known as Uncented transform is used. When there is sever non-linearity, 
Signal \& Image Processing : An International Journal (SIPIJ) Vol.8, No.6, December 2017

both EKF and UKF cannot handle the issue. The particle filter is a complete non-linear state estimator is used to address the issues of sever nonlinearity.

\section{REFERENCES}

[1] K. H and M. Viberg, "Two decades of array signal processing research aparametric approach," IEEE Signal Process. Mag. Vol13 No4 Pp 67-94, 1996.

[2] "Satish chandran Advances in Direction of Arrival Estimation Artech House,London,2010

[3] E. Brookner, Tracking and Kalman Filtering Made Easy. John Wiley and sons, 1998.

[4] R.Schmidt, "Multiple Emitter Location and Signal Parameter Estimation," IEEE Trans Antennas Propag. Vol AP-34, Mar. 1986.

[5] Y. T. S. N. Jagadeesha and G. L.J, "Expectation maximization- matrix pencil method for direction of arrival estimation.," Proc 7th Int. Symp. Commun. Syst. Netw. Digit. Signal Process. CSNDSP Pp 9195, 2010.

[6] Yerriswamy.T, "Direction of arrival estimation using array signal processing techniques," Faculty of computer science and engineering, VTU, India, August2012.

[7] R. E. Kalman, "A new approach to linear filtering and prediction problems," J. Basic Eng., vol. 82, no. 1, pp. 35-45, 1960.

[8] J. S. N, "A Comparative Study of Adaptive Algorithms with Applications toBeamforming," Department of Electronics and Computer Engineering, University of Roorkee, Roorkee, India, 1994.

[9] S. Haykin, Adaptive filters. Pearson Education pvt ltd, 2011.

[10] S. J. Julier and J. K. Uhlmann, "Unscented filtering and nonlinear estimation," Proc. IEEE, vol. 92, no. 3, pp. 401-422, Mar. 2004.

[11] K. Okuma, A. Taleghani, N. De Freitas, J. J. Little, and D. G. Lowe, "A boosted particle filter: Multitarget detection and tracking," in European conference on computer vision, 2004, pp. 28-39.

[12] BenjaminFriedlander, "The root-MUSIC algorithm for direction finding with interpolated arrays," Signal Process. Elsevier, 1993.

[13] R. Roy and T. Kailath, "ESPRIT - Estimation of Signal Parameters via Rotational Invariance Techniques,” IEEE Trans ASSP Vol 37 No7 Pp 984-995, 1989.

[14] C. R. S. C.R.Rao and B.Zhou, "Tracking the direction of arrival of multiple moving targets," Trans IEEE Signal Process. Vol 42 No 5, May 1994.

[15] M. S. Arulampalam, S. Maskell, N. Gordon, and T. Clapp, "A tutorial on particle filters for online nonlinear/non-Gaussian Bayesian tracking,” IEEE Trans. Signal Process., vol. 50, no. 2, pp. 174-188, Feb. 2002.

[16] M. H. Hayes, Statistical Digital Signal Processing and Modelling. John Wiley and sons, 1996.

[17] P.A.Thompson, "An adaptive spectral analysis technique for unbiased frequency estimation in the presence of the white noise," Proc. 13thAsilomar Conf. Circuit Syst. Comput. Pac. Grove CA, 1980.

[18] P. C. \& G. H. Golub, "Tracking a few extreme singular values and vectors in signal processing," Proc. IEEE.

[19] G. H. Golub and C. F. V. Loan, Matrix Computations. Hindustan Book agency, 2007. 
Signal \& Image Processing : An International Journal (SIPIJ) Vol.8, No.6, December 2017

[20] P. Del Moral and L. M. Murray, "Sequential Monte Carlo with Highly Informative Observations," ArXiv14054081 Stat, May 2014.

[21] P. M. Djuric et al., "Particle filtering," IEEE Signal Process. Mag., vol. 20, no. 5, pp. 19-38, Sep. 2003.

[22] J. S. Liu and R. Chen, "Sequential Monte Carlo methods for dynamic systems," J. Am. Stat. Assoc., vol. 93, no. 443, pp. 1032-1044, 1998.

\section{AUTHORS}

Venu Madhava.M received his B.E.,in Instrumentation Technology and M.Tech., in Industrial Electronics, from Visvesvaraya Technological University, Belgaum, Karnataka India in 2004 and 2009 respectively. He is currently working towards his Doctoral Degree from Visvesvaraya Technological University, Belgaum, Karnataka, India. At present he is working as Assistant Professor, in the Department of Electronics and Communication,Proudhadevaraya Institute of Technology,(Affiliated to Visvesvaraya Technological University), Hospet, Karnataka, India.

Dr. S. N. Jagadeesha received his B.E., in Electronics and Communication Engineering, from University B. D. T College of Engineering., Davangere affiliated to Mysore University, Karnataka, India in 1979, M..E. from Indian Institute of Science (IISC), Bangalore, India specializing in Electrical Communication Engineering., in 1987 and Ph.D. in Electronics and Computer Engineering., from University of Roorkee (I.I.T, Roorkee), Roorkee, India in 1996. He is an IEEE member and Fellow, IETE. His research interest includes Array Signal Processing, Wireless Sensor Networks and Mobile Communications. He has published and presented many papers

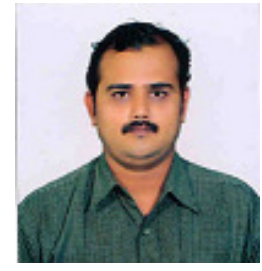
on Adaptive Array Signal Processing and Direction-of-Arrival estimation. Currently he is Professor and Head in the Department of Computer Science and Engineering, PESITM. (Affiliated to Visvesvaraya Technological University), Shimoga, Karnataka, India.

Dr.Yerriswamy received his B.E. in Electronics and Communication Engineering, From RYMEC, Bellary affiliated to Gulbarga University, Gulbarga, Karnataka, India in 2000,M.Tech in Network and Internet Engineering from JNNCE, Shimoga, Affiliated to Visvesvaraya Technological University, Belgaum, India in 2005 and $\mathrm{Ph} . \mathrm{D}$ in the Faculty of Computer and Information Science form Visvesvaraya Technological University, Belgaum, Karnataka, India He is a member of ISTE. His research interests includes Array Signal Processing, Wireless Sensor Networks, Cognitive Radios. He has published many papers in Direction-of-Arrival estimation

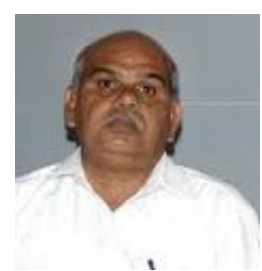
and Array signal processing. Currently he is Professor and Head in the Department of Computer science and Engineering, KLE Institute of Technology, (Affiliated to Visvesvaraya Technological University, Belgaum), Hubli, Karnataka, India 